\title{
Disseminated Cryptococcosis in a Deceptively Immunocompetent Adolescent
}

\section{Vivek Sood ${ }^{1 *}$, Raja Ramachandran ${ }^{1}$, Rakesh Kumar Pilania ${ }^{2}$, Arun Prabhakar ${ }^{3}$, Neeraj Inamdar', Navin Pattanashetti ${ }^{1}$, Vibhu Joshi ${ }^{2}$, Madhubala Sharma ${ }^{2}$, Amit Rawat ${ }^{2}$, HS Kohli ${ }^{1}$ and KL Gupta ${ }^{1}$}

\author{
${ }^{1}$ Department of Nephrology, Postgraduate Institute of Medical Education and Research, India \\ ${ }^{2}$ Allergy Immunology Unit, Department of Pediatrics, Advanced Pediatrics Centre, Postgraduate \\ Institute of Medical Education and Research, India \\ ${ }^{3}$ Department of Internal Medicine, Postgraduate Institute of Medical Education and Research, India
}

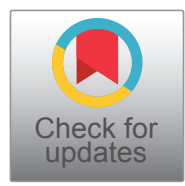

*Corresponding author: Dr. Vivek Sood, Department of Nephrology, Postgraduate Institute of Medical Education and Research (PGIMER), Chandigarh - 160012, India, Tel: 0897-4321-019, Fax: +91-172-2744401

\begin{abstract}
Cryptococcosis particularly if disseminated or extrapulmonary, in an otherwise immune-competent individual, especially if young, requires thorough evaluation for underlying primary immunodeficiency including Mendelian susceptibility to mycobacterial disease (MSMD). In this context, we describe an adolescent diagnosed with disseminated cryptococcosis involving skin, lymph nodes, spleen and hepato-biliary involvement with reduced IL-12 $\beta 1$ receptor expression suggesting underlying MSMD. Following initial management with conventional amphotericin and flucytosine, along with antibiotics for cholangitis and later suppressive prophylaxis with fluconazole our patient showed good response with resolution of jaundice, normalization of liver function tests by second month of follow-up and had significant regression of lymph nodes as well as skin lesions by six months.
\end{abstract}

\section{Keywords}

MSMD, Disseminated cryptococcosis

\section{Introduction}

Disseminated cryptococcosis is most commonly associated with HIV infection. However, cryptococcal infection in an apparently immunocompetent individual, requires exclusion of underlying primary immunodeficiency. Mendelian susceptibility to mycobacterial disease (MSMD) is a rare innate immune defect characterized by predisposition to infections by less virulent mycobacteria, non-typhoidal salmonellosis and more rare- ly with other intra-macrophagic fungi or parasites [1]. In this context, we describe an adolescent diagnosed with disseminated cryptococcosis involving skin, lymph nodes, spleen and hepato-biliary involvement with reduced IL-12 $\beta 1$ receptor expression.

\section{Case Summary}

A sixteen-years-old laborer, with non-contributory past history, presented with fever, jaundice and unintentional weight loss of eight kilograms over two months. The patient had also noticed development of new lesions over his face and trunk. Clinically patient was thin build (BMI-16.4 kg/m²), febrile $\left(39^{\circ} \mathrm{C}\right)$, had non-tender, generalised lymphadenopathy (cervical, axillary and inguinal), warty plaques over face and trunk (Figure 1) with hepato-splenomegaly. Investigations revealed haemoglobin-6.4 g/L, leukocytosis (11.4 $\times 10^{\circ} / \mathrm{L}-94 \%$ neutrophils) and normal platelets. Viral markers including HIV-1 and 2 were negative with CD4 count of 564 cells $/ \mathrm{mm}^{3}$. Tuberculin sensitivity test was non-reactive $(0 \mathrm{~mm})$. Lymph node biopsies (cervical and inguinal) showed granulomatous reaction and Alcian blue stain revealed blue coloured capsule consistent with cryptococcosis. Further Ziehl Neelsen stain for acid-fast bacilli, mycobacterial PCR and gene Xpert were negative from the specimen. Biopsy from skin lesion also revealed cryptococcal infection and blood was incubated in BACTEC aerobic and anaerobic culture bot-

Citation: Sood V, Ramachandran R, Pilania RK, Prabhakar A, Inamdar N, et al. (2019) Disseminated Cryptococcosis in a Deceptively Immunocompetent Adolescent. Int J Rare Dis Disord 2:013. doi. org/10.23937/2643-4571/1710013

Accepted: October 26, 2019; Published: October 28, 2019

Copyright: (C) 2019 Sood V, et al. This is an open-access article distributed under the terms of the Creative Commons Attribution License, which permits unrestricted use, distribution, and reproduction in any medium, provided the original author and source are credited. 


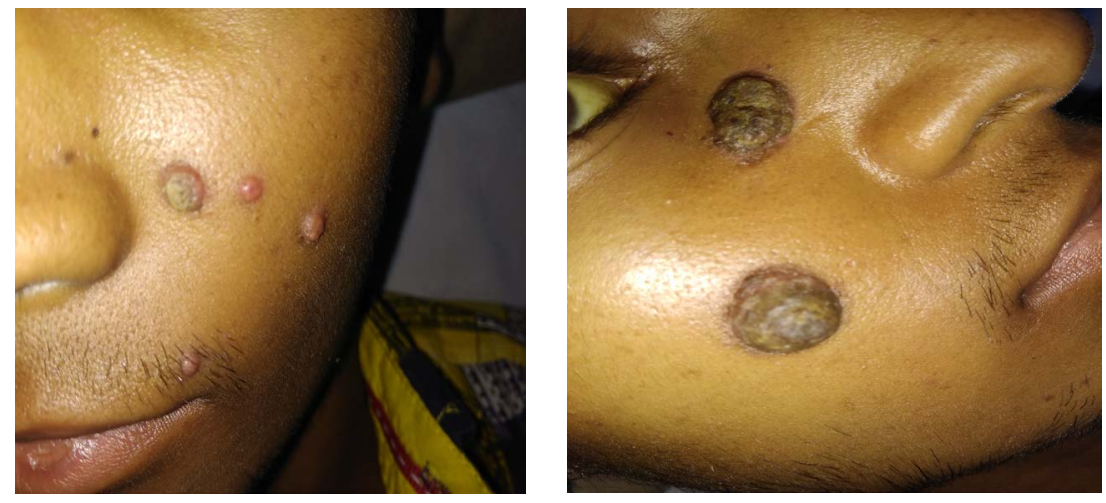

Figure 1: Warty plaque like lesions over the face.

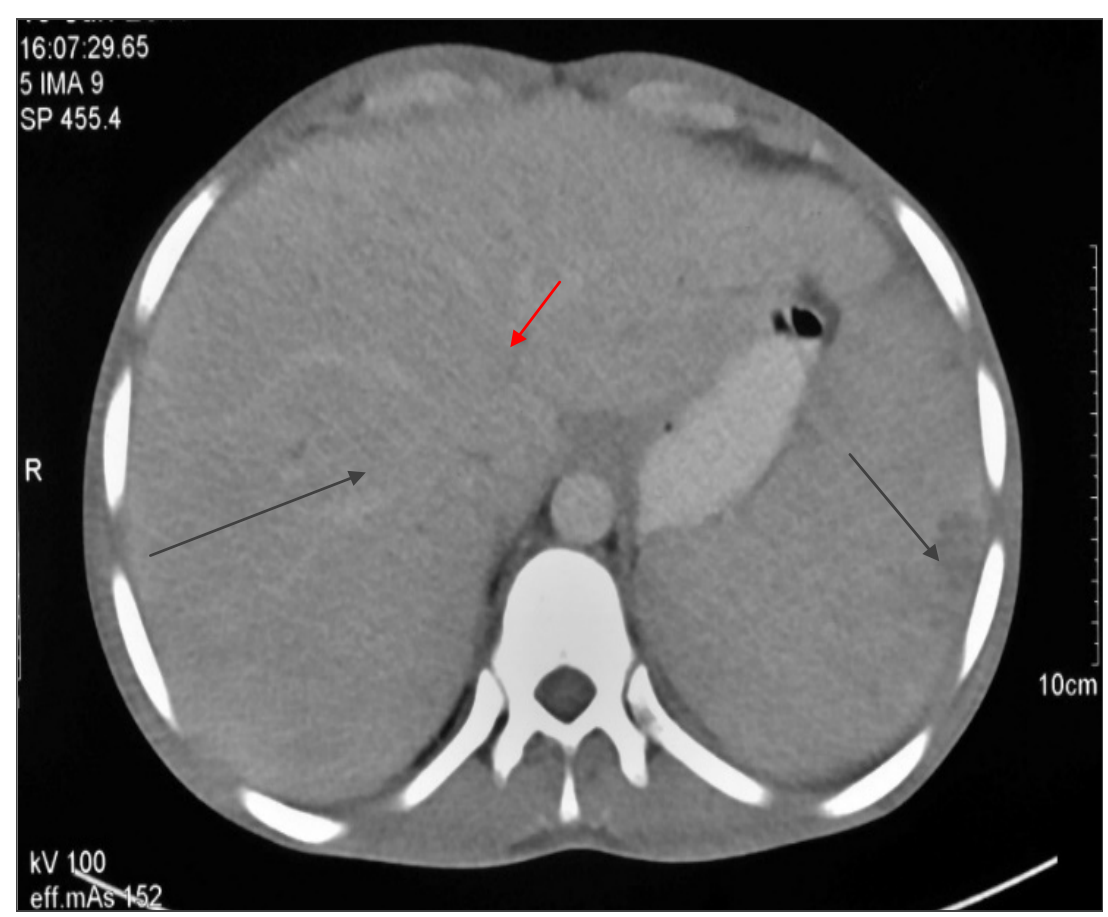

Figure 2: CECT abdomen showing multiple hypoechoic lesions in liver and spleen (black lines) along with intrahepatic biliary duct dilatation (red line).

tles. After five days of incubation, growth was noticed and subsequent sub-cultures were done on blood agar, chocolate agar and Mac Conkey agar. After two days of inoculation, colonies from blood agar revealed gram positive round budding yeast cells. Colonies were further sub-cultured on Saboraud's dextrose agar, Christensen's urea and birdseed agar. Colour of the slant in Christensen's media turned to magenta in one day establishing urease production by the organism and demonstration of brownish colonies on birdseed agar, further established characteristic phenol oxidase production by Cryptococcus neoformans. Patient also had obstructive jaundice (total and conjugated bilirubin of $10 \mathrm{mg} / \mathrm{dL}$ and $7.8 \mathrm{mg} / \mathrm{dL}$ respectively) with raised ALP (1954 IU/L) and GGT (358 U/L). Contrast enhanced CT showed generalized (mediastinal, abdominal, inguinal) lymphadenopathy, intrahepatic biliary duct dilatation, hepato-splenomegaly with multiple hypoechoic lesions (Figure 2). Initially jaundice was attributed to obstruc- tion by peri-portal lymphadenopathy, however endoscopic ultrasound and MRCP suggested sclerosing cholangitis and consequent autoimmune hepatitis workup (including ANA, AMA and ASMA) was negative. Further immunodeficiency work-up including immunoglobulin profile, Nitro Blue tetrazolium test, T and B lymphocyte counts and NK cell counts were normal for age. Flow cytometry for expression of IL12R $\beta 1$ was performed on peripheral blood mononuclear cells following stimulation with phytohemagglutinin and culture for 72 hours. Expression of IL12R 31 on gated CD3 + T lymphocytes was reduced compared to control (Figure 3). Targeted next-generation sequencing was performed using a custom designed gene panel comprising of 44 common primary immunodeficiency disease genes which include four genes (IFNGR1, IFNGR2, IL12RB1 and STAT1) associated with MSMD phenotype on the IONS5 platform from Thermo Fisher Scientific. No pathogenic variants were found in IL12RB1 or other genes associated with 


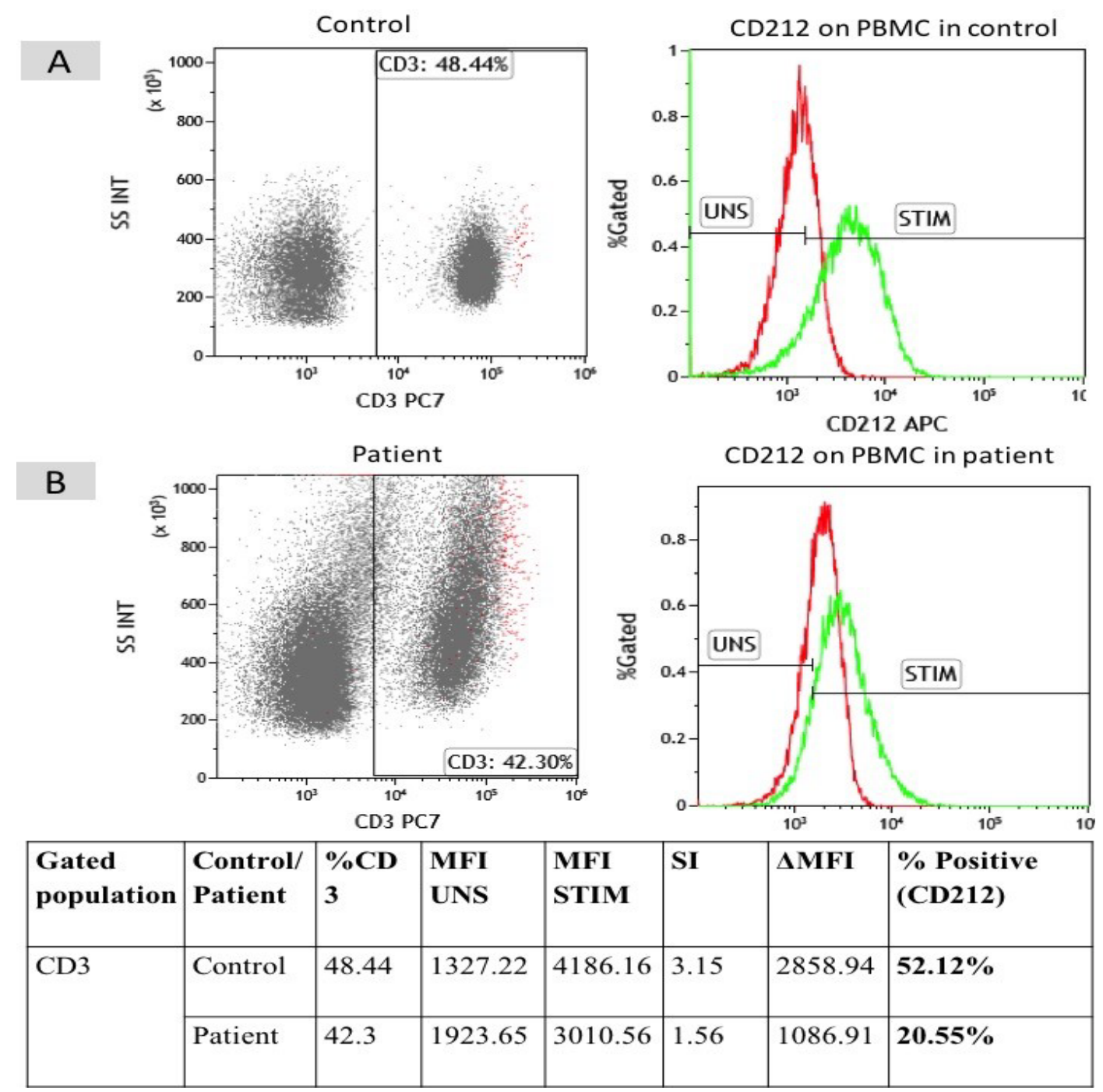

Figure 3: Flow cytometry for expression of IL12R $\beta 1$ on peripheral blood mononuclear cells (PBMC) following stimulation with phytohemagglutinin and culture for $72 \mathrm{hrs}$. The expression of IL12R 1 on gated CD3+ T lymphocytes was reduced in index patient (Figure 1A) compared to control (Figure 1B) (SI Index patient: 1.5 Control: 3.1, percent positivity; $20.5 \%$ index patient; Control: $52.12 \%)$.

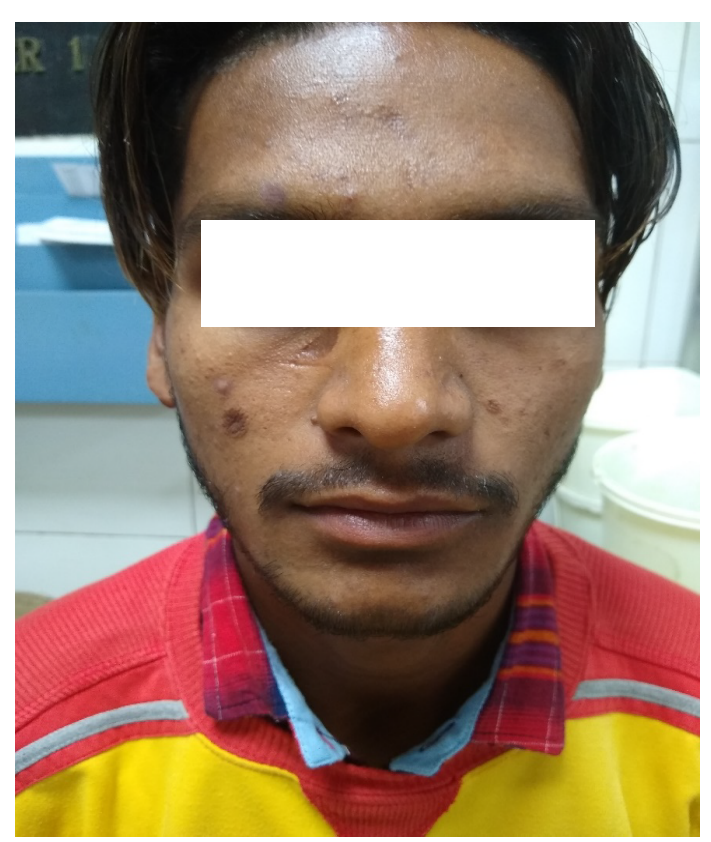

Figure 4: Near complete resolution of warty plaque like lesions over the face following anti-fungal therapy.
MSMD. All exons of the IL12RB1 were well covered and no deletion (homozygous or heterozygous) were noted. Patient was initially managed with conventional amphotericin (1 mg/Kg; cumulative $4.5 \mathrm{~g}$ over 6 weeks) and flucytosine $(25 \mathrm{mg} / \mathrm{Kg})$, along with antibiotics for cholangitis and later switched over to suppressive prophylaxis with fluconazole. His jaundice improved following anti-fungal therapy and liver function tests normalized by second month of follow-up and lymph nodes as well as skin lesions (Figure 4) also had regressed significantly by six months.

\section{Discussion}

IL-12-IFN- $\gamma$ pathway [2], required for activation of $T$ cells and NK cells, is defective in MSMD, making the individual susceptible for infection from intracellular pathogens. Fungal infections in MSMD include candidiasis, histoplasmosis, coccidioidomycosis and Paracoccidioidomycosis.

Cryptococcosis is infrequently reported $[3,4]$ and 
even if there is tangible isolated organ involvement by cryptococcus, thorough evaluation for possible dissemination requires consideration as in index case who had metastatic dissemination to lymph nodes, skin, hepato-biliary system and spleen (without brain or bone marrow involvement) which has never been described in MSMD.

Liver involvement include focal granulomas, regenerative nodules, sclerosing cholangitis [5]; all were present in index patient.

Virulence of $C$. neoformans is consequent to polysaccharide capsule [6] which can be visualized with light microscopy using India ink, mucicarmine or Periodic acid Schiff (PAS) stain with high specificity (97\%) but poor sensitivity (73\%) [7]. Etiology of Cryptococcus neoformans in index case was established on the basis of phenotypic and biochemical characteristics alone and techniques such as PCR and MALDI-TOF couldn't be done due to financial constraints.

The in vitro activity of antifungal agents was determined by the Etest (AB Biodisk, Solna, Sweden) in accordance with the manufacturer's instructions. MICs for the isolate were: Amphotericin B - $0.250 \mu \mathrm{g} / \mathrm{mL}$, Fluconazole - $2 \mu \mathrm{g} / \mathrm{mL}$, Itraconazole - $0.250 \mu \mathrm{g} / \mathrm{mL}$, voriconazole $-0.1250 \mu \mathrm{g} / \mathrm{mL}$.

Target next generation sequencing did not reveal any pathogenic variants. However, deeper intronic variants which may be pathogenic are not detected by this method and require a whole genome sequencing (WGS) of the patient along with parents. Other family members of the index patient were not affected, nevertheless WGS could not be performed due to financial constraints. Likewise phenocopies of genetic defects in IFNGR1, IFNGR2, IL12RB1, IL12RB2, IL12A (p35 subunit), IL12B (p40 subunit) due to autoantibodies to IFN $\gamma$, IL12p35 or IL12p40 have also been reported to be associated with MSMD however, could not be tested owing to financial constraints. The IL12RB1 gene is enriched for alu repeat sequences $(44.7 \%$ compared to $25.8 \%$ for Chromosome 19 and $10.5 \%$ of the entire human genome). Enrichment for these alu repeats in the IL12RB1 gene predisposes to genomic instability leading to significant increase in copy number variations [8].

\section{Conclusion}

MSMD is a rare entity signifying an apparent late presentation of congenital immunodeficiency. It predisposes to infections by less virulent intra-macrophagic organisms; treatment guidelines for which are still lacking; though good response has been observed following long term appropriate anti-microbial therapy; duration of which is still debatable.

\section{References}

1. Bustamante J, Boisson-Dupuis S, Abel L, Casanova JL (2014) Mendelian susceptibility to mycobacterial disease: Genetic, immunological, and clinical features of inborn errors of IFN-y immunity. Semin Immunol 26: 454-470.

2. Retini C, Kozel TR, Pietrella D, Monari C, Bistoni F, et al. (2001) Interdependency of interleukin-10 and interleukin-12 in regulation of $\mathrm{T}$-cell differentiation and effector function of monocytes in response to stimulation with Cryptococcus neoformans. Infect Immun 69: 6064-6073.

3. Rezai MS, Khotael G, Kheirkhah M, Hedayat T, Geramishoar M, et al. (2008) Cryptococcosis and deficiency of interleukin12r. Pediatr Infect Dis J 27: 673.

4. Jirapongsananuruk $O$, Luangwedchakarn $\mathrm{V}$, Niemela JE, Pacharn P, Visitsunthorn N, et al. (2012) Cryptococcal osteomyelitis in a child with a novel compound mutation of the IL12RB1 gene. Asian Pac J Allergy Immunol 30: 79-82.

5. Bucuvalas JC, Bove KE, Kaufman RA, Gilchrist MJ, Oldham KT, et al. (1958) Cholangitis associated with cryptococcus neoformans. Gastroenterology 88: 1055-1059.

6. Lan SH, Chang WN, Lu CH, Lui CC, Chang HW (2001) Cerebral infarction in chronic meningitis: A comparison of tuberculous meningitis and cryptococcal meningitis. QJM 94: 247-253.

7. Saha DC, Xess I, Biswas A, Bhowmik DM, Padma MV (2009) Detection of cryptococcus by conventional, serological and molecular methods. J Med Microbiol 58: 10981105.

8. Rosain J, Oleaga-Quintas C, Deswarte C, Verdin H, Marot $S$, et al. (2018) A variety of Alu-mediated copy number variations can underlie IL-12Rß1 deficiency. J Clin Immunol 38: 617-627. 